\title{
Spontaneous Resolution of Cataract and Medical Management of Corneal Opacity in an Indian Parrot-Psittacula krameri manillensis
}

\author{
Sarbani Hazra \\ Department of Veterinary Surgery \& Radiology, West Bengal University of Animal \& Fishery Sciences, Kolkata, India \\ Email: shazrakon@yahoo.co.in
}

Received March 24, 2013; revised April 22, 2013; accepted May 22, 2013

Copyright (c) 2013 Sarbani Hazra. This is an open access article distributed under the Creative Commons Attribution License, which permits unrestricted use, distribution, and reproduction in any medium, provided the original work is properly cited.

\begin{abstract}
A 35-year-old Indian parrot (Psittacula krameri manillensis) was presented to the Department of Veterinary Surgery \& Radiology, West Bengal University of Animal and Fishery Sciences with a history of blepharospasm and corneal lesion OS. Test with fluorescein dye was negative. The dense corneal opacity (macula) was identified as involving the posterior corneal layer. The further ophthalmic examination was done and hypermature phacolytic cataract was diagnosed. No other abnormality was detected. Medical management with topical nepafenac prednisolone and triple antibiotic was instituted. The corneal lesion subsided completely within one week followed by spontaneous resorption of the cataract. The treatment protocol was successfully eliminated the discomfort and intraocular lesions in the senile parrot.
\end{abstract}

Keywords: Medical Management; Senile Cataract; Parrot

\section{Introduction}

It has been reported that cataract is a frequent occurrence in birds [1]. Senility has been recorded as a major cause of cataract in birds [2,3]. Detection of cataract in birds has been largely from necropsy findings. We present an interesting clinical case report of senile cataract with associated corneal opacity in an Indian Parrot, Psittacula krameri manillensis and its successful medical management. There has been no published report of spontaneous resorption of senile hyper mature cataract in an Indian parrot so far.

\subsection{History and Ophthalmic Examination}

A 35-year-old parrot, Psittacula krameri manillensis was presented to the Dept. of Veterinary Surgery \& Radiology, West Bengal University of Animal and Fishery Sciences with history of blepharospasm and corneal lesion OS. The affected eye was negative to fluorescein dye test. Detailed ophthalmic examination of both eyes was performed with an ophthalmoscope, slit lamp biomicroscope and indirect ophthalmoscope. A dense corneal opacity (macula) was identified as involving the posterior corneal layer OS (Figure 1A). Hypermature phacolytic cataract was diagnosed OS (Figure 1A). No other ab- normality was detected in either eye.

\subsection{Medical Treatment}

It was decided to initiate medicinal therapy for treating the corneal opacity, and then consider the possibility of extracting the cataractous lens. The bird was prescribed topical instillation of nepafenac (Nevanac ${ }^{\circledR}$, Alcon) thrice daily, prednisolone eye drop thrice daily and triple antibiotic twice daily for 7 days. The owner was advised to return for recheck every week.

\subsection{Follow up}

The corneal opacity had completely resolved after one week (Figure 1B). Another interesting observation was made while doing a detailed ophthalmic examination, the cataract had begun to resolve (Figure 1B). The blepharospasm was absent and the bird appeared more comfortable. The owner was advised to continue with the prescribed medicines for another three weeks, it was also explained that the surgery may not be required for removal of cataract as there was an indication of spontaneous resorption.

On subsequent follow up examination, the cataract was observed to be gradually resolved (Figures 1C-E) and 


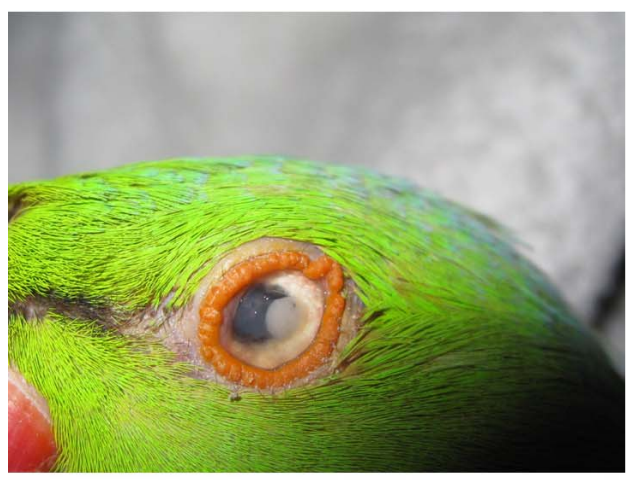

A

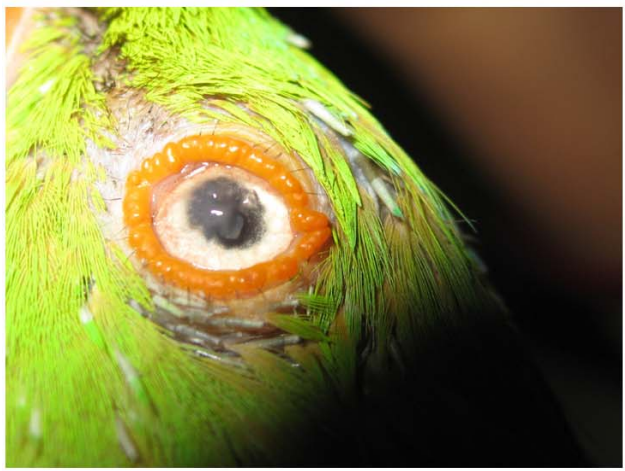

C

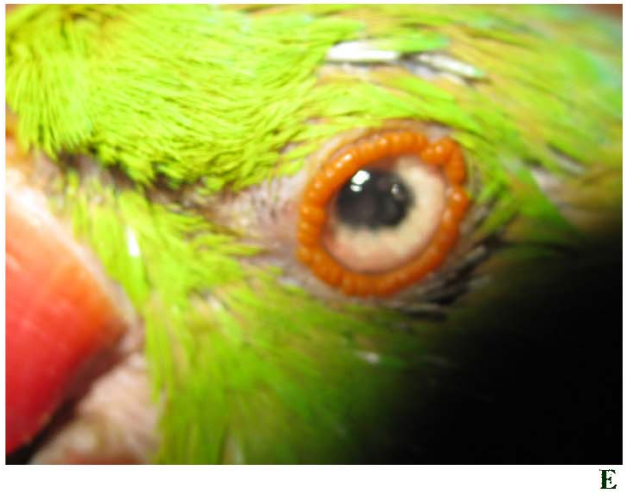

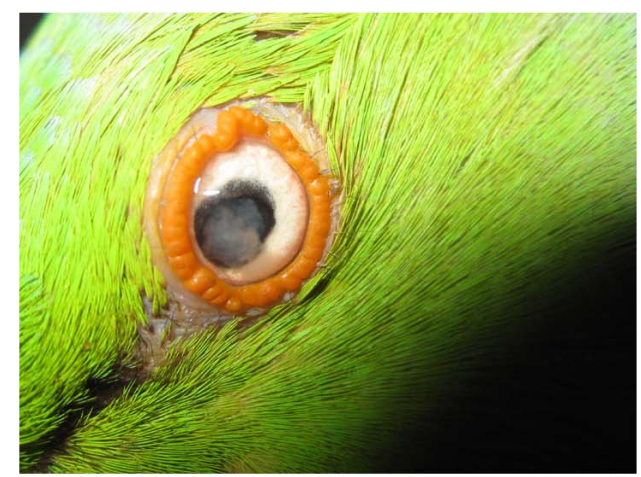

B

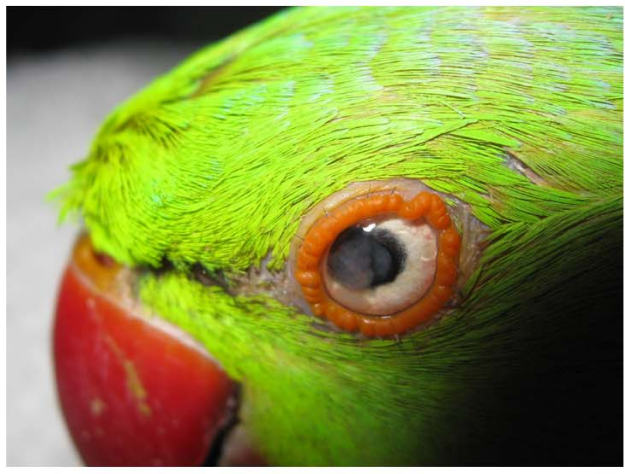

D

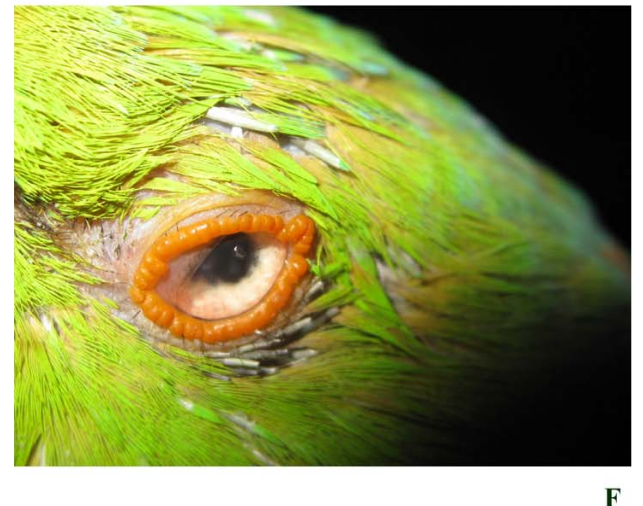

F

Figure 1. Figure shows the case of corneal opacity and cataract in a parrot (Figure 1A), the treatment regimen cleared the corneal opacity within one week (Figure 1B), subsequently the cataract was observed to be resolved gradually (Figures 1C-E), resulting in complete cure within three weeks (Figure 1F).

after three weeks it resolved completely. The bird was cured at the end of three weeks following the medicinal treatment (Figure 1F).

\section{Discussion}

A number of literatures report incidence of cataract in birds [4-7]. In the present clinical case, the identification of the disease condition was followed by successful treatment of the condition by medicinal therapy. In this patient, hyper mature cataract progressed to liquifactive changes, the corneal opacity observed was assumed to be due to lens induced uveitis. Existing literature suggested preferring conservative therapy for lens induced uvietis to surgical treatment [1]. The patient responded well to the topical treatment with nepafenac, a potent cox2 inhibitor and topical prednisolone. Complete regression of the corneal lesion was seen following one week of treatment. A broad spectrum antibiotic was initiated as prophylaxis against infection. This is the first report of ocular use of nepafenac in birds. No adverse effect was noted with the use of the drug. Spontaneous resorption of cataracts has been first time reported in Great horned owl and Screech owls [7], the birds were young and resorption occurred over a period of three months to one year. In our case it was a senile cataract, the bird was 35 years old and the resorption was complete by three weeks. 
There may be some species variation or there could be a possible role of the medicinal therapy for the early resorption. This may be investigated further.

This is the first report of spontaneous resorption of hypermature senile cataract with associated corneal opacity in a parrot which was successfully treated with medicinal therapy.

\section{REFERENCES}

[1] J. M. Nicholas, "Exotic Animal Ophthalmology,” In: K. N. Gelatt, Ed., Veterinary Ophthalmology, 2nd Edition, Lea and Febiger, Philadelphia, 1991, pp. 680-705.

[2] I. F. Keymer, “Cataract in birds,” Avian Pathology, Vol. 6, No. 4, 1977, pp. 335-341. doi:10.1080/03079457708418243
[3] T. J. Kern, C. J. Murphy and R. C. Riis, "Lens Extraction by Phacoemulsification in Two Raptors," Journal of the American Veterinary Medical Association, Vol. 185, No. 11, 1984, pp. 1403-1406.

[4] S. L. Clubb and L. G. Karpinski, “Aging in Macaws,” Journal of the Association of Avian Veterinarians, Vol. 7, No. 1, 1993, pp. 31-33. doi:10.2307/30134306

[5] M. Jahne, "Cataract in a Parrot,” Monatshefte fur Veterinarmedizin, Vol. 34, No. 15, 1979, pp. 584-585.

[6] R. E. Schmidt, "Hyper Mature Cataract in a Crested Mynah, Leucopsar Rothschildi,” Journal of Wildlife Diseases, Vol. 19, No. 2, 1983, pp. 158-159.

[7] N. C. Buyukmihci, C. J. Murphy and T. Schulz, "Developmental Ocular Diseases of Raptors," Journal of Wildlife Diseases, Vol. 24, 1988, p. 207. 